\title{
Correlation of Large Longitudinal Deformations With Different Strain Histories
}

\author{
L. J. Zapas and T. Craft
}

(July 29, 1965)

\begin{abstract}
It is shown that the BKZ incompressible elastic fluid theory is in excellent agreement with experimental results obtained in simple extension. From single step stress-relaxation data, the stress-strain response for a number of other simple extension histories are calculated from the theory and are compared with experiments.
\end{abstract}

Key Words: BKZ theory, constant rate of strain, creep, elastic fluid, nonlinear behavior, polyisobutylene, polyvinylchloride, recovery, stress relaxation.

\section{Introduction}

In 1963 Bernstein, Kearsley, and Zapas [1] ${ }^{1}$ presented a theory of an elastic fluid which gave the correct stress-relaxation response for a large variety of elastomeric materials, including vulcanized rubbers. A principle attractiveness of this theory is its relative simplicity; with a single integral in time, it describes the stress-strain behavior for all types of deformation histories. In the case of simple extension; it predicts the behavior in any uniaxial strain history from the results of single step stress-relaxation experiments which cover the same range of extension and time. We designed a series of experiments to check the validity of this theory and found, as is shown in section 4 of this paper, excellent agreement with experiment in all cases. We are aware that experiments cannot prove a theory. From our results, however, we feel strongly that a single integral expression with a nonlinear integrand such as the BKZ elastic fluid equation is sufficient to describe the stress-strain behavior of elastomeric materials.

\section{General Considerations}

For an extensive description of the BKZ elastic fluid, we refer the reader to the initial papers [1,2].

Here we shall use only the definitions and derivations necessary for the description of uniaxial deformations, i.e., simple extension of an incompressible material.

A particular motion of the material may be specified by a relation between the Cartesian components of the position of a particle at time $t, x_{i}(t)$ and of its position at time $\tau, x_{i}(\tau)$,

$$
x_{i}(t)=x_{i}\left(x_{k}(\tau), t, \tau\right) \quad i, k=1,2,3 .
$$

\footnotetext{
' Figures in brackets indicate the literature references at the end of this paper
}

The relative deformation gradients $x_{i k}(t, \tau)$ are defined by

$$
x_{i k}(t, \tau)=\frac{\partial x_{i}(t)}{\partial x_{k}(\tau)} .
$$

The left Cauchy-Green tensor $c_{i j}(t, \tau)$ is defined by

$$
c_{i j}(t, \tau)=x_{i k}(t, \tau) x_{j k}(t, \tau)
$$

where, in accordance with Cartesian tensor notation, repeated indices indicate summation over the values $1,2,3$. The principal invariants of $c_{i j}(t, \tau)$ are

$$
\begin{aligned}
& I_{1}(t, \tau)=\xi_{1}^{2}+\xi_{2}^{2}+\xi_{3}^{2} \\
& I_{2}(t, \tau)=\xi_{1}^{2} \xi_{2}^{2}+\xi_{2}^{2} \xi_{3}^{2}+\xi_{3}^{2} \xi_{1}^{2} \\
& I_{3}(t, \tau)=\xi_{1}^{2} \xi_{2}^{2} \xi_{3}^{2}
\end{aligned}
$$

where $\xi_{1}=\frac{\lambda_{1}(t)}{\lambda_{1}(\tau)}, \xi_{2}=\frac{\lambda_{2}(t)}{\lambda_{2}(\tau)}$ and $\xi_{3}=\frac{\lambda_{3}(t)}{\lambda_{3}(\tau)}$.

In view of the condition of incompressibility which requires that $I_{3}(t, \tau)=1$, the constitutive equation for the BKZ elastic fluid can be written

$\sigma_{i j}=-p \delta_{i j}+2 \int_{-\infty}^{t}\left[\frac{\partial U}{\partial I_{1}} c_{i j}(t, \tau)-\frac{\partial U}{\partial I_{2}} c_{i j}^{-1}(t, \tau)\right] d \tau$

where $\sigma_{i j}$ are the components of the stress tensor, $p$ is some hydrostatic pressure, $U$ is a function of $I_{1}, I_{2}$, and $t-\tau$,

$$
U=U\left(I_{1}(t, \tau), I_{2}(t, \tau), t-\tau\right)
$$

and $c_{i j}^{-1}(t, \tau)$ are the components of the inverse of $c_{i j}(t, \tau)$.

We may describe isochoric uniaxial strain in a coordinate system in which the $x_{1}$ direction corresponds to the direction of stretch. Then the particle which in some reference configuration is located at $X_{1}, X_{2}, X_{3}$ 
has a position at time $t, x(t)$ given by

$$
\begin{aligned}
& x_{1}(t)=\lambda(t) X_{1} \\
& x_{2}(t)=\lambda^{-1 / 2}(t) X_{2} \\
& x_{3}(t)=\lambda^{-1 / 2}(t) X_{3} .
\end{aligned}
$$

The matrix of the left Cauchy-Green tensor $c_{i j}(t, \tau)$ becomes

$$
\left\|c_{i j}(t, \tau)\right\|=\left\|\begin{array}{ccc}
\frac{\lambda^{2}(t)}{\lambda^{2}(\tau)} & 0 & 0 \\
0 & \frac{\lambda(\tau)}{\lambda(t)} & 0 \\
0 & 0 & \frac{\lambda(\tau)}{\lambda(t)}
\end{array}\right\|
$$

and

$$
\begin{aligned}
& I_{1}(t, \tau)=\frac{\lambda^{2}(t)}{\lambda^{2}(\tau)}+2 \frac{\lambda(\tau)}{\lambda(t)} \\
& I_{2}(t, \tau)=2 \frac{\lambda(t)}{\lambda(\tau)}+\frac{\lambda^{2}(\tau)}{\lambda^{2}(t)} .
\end{aligned}
$$

From (1), (2), and (3) we get

$$
\begin{aligned}
& \sigma(t)=\sigma_{11}(t)-\sigma_{22}(t) \\
& =\int_{-\infty}^{t}\left[\frac{\lambda^{2}(t)}{\lambda^{2}(\tau)}-\frac{\lambda(\tau)}{\lambda(t)}\right] h\left(\frac{\lambda(t)}{\lambda(\tau)}, t-\tau\right) d \tau
\end{aligned}
$$

where

$$
h\left(\frac{\lambda(t)}{\lambda(\tau)}, t-\tau\right)=2\left(\frac{\partial U}{\partial I_{1}}+\frac{\lambda(\tau)}{\lambda(t)} \frac{\partial U}{\partial I_{2}}\right) .
$$

Thus, if we know the function $h$, we can predict the stress response to an uniaxial strain history. ${ }^{2}$ For a single step stress relaxation, for which $\lambda(t)=1$ for $t<0$ and $\lambda(t)=\lambda$, a constant, for $t \geqslant 0$, eq (4) becomes

$$
\sigma(t)=\left(\lambda^{2}-\frac{1}{\lambda}\right) H(\lambda, t)
$$

where

$$
H(\lambda, t)=\int_{t}^{\infty} h(\lambda, \zeta) d \zeta
$$

and

$$
h(\lambda, t)=-\frac{\partial H(\lambda, t)}{\partial t}
$$

${ }^{2}$ For discussion of other strain histories we refer the reader to a recent paper by Bernstein $[3 \mid$.

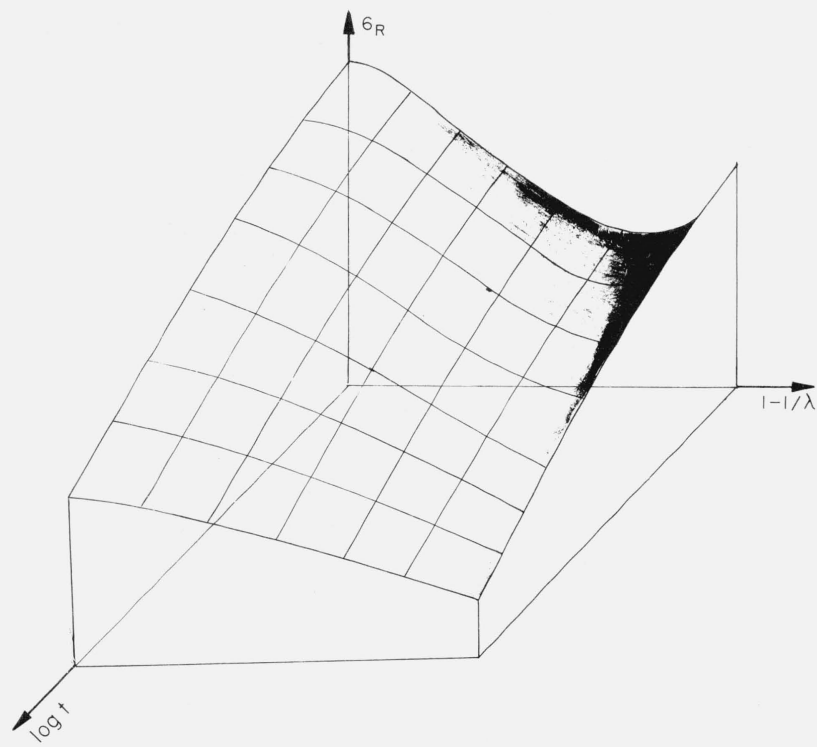

FigURE 1. A representative three dimensional plot of $\sigma_{\mathrm{R}}=\mathrm{H}(\lambda, \mathrm{t})$.

In their 1963 article Bernstein, Kearsley, and Zapas [1] plotted isochrones of a reduced stress $\sigma_{R}(\lambda, t)$ which they defined by

$$
\sigma_{R}(\lambda, t)=\frac{\sigma(t)}{\lambda^{2}-\frac{1}{\lambda}} .
$$

It is evident from (5) that $\sigma_{R}(\lambda, t)$ is identical with $H(\lambda, t)$. A typical three dimensional plot of $H$ versus $\log$ time and $l / \lambda$ is the surface shown in figure 1 . We see, then, that with the stress histories of single step uniaxial strains we can measure $H(\lambda, t)$ and predict the stress history of any uniaxial strain history.

\section{Experimental Procedure}

All our experiments in this work deal with uniaxial deformations on strips with rectangular cross sections. These strips measured about $17 \mathrm{~cm}$ long, $0.88 \mathrm{~cm}$ wide, and $0.21 \mathrm{~cm}$ thick. The data were obtained on polyisobutylene VISTANEX L-100 samples and on polyvinyl chloride samples (plasticized with 50 percent by weight of tricresol phosphate). The stress-relaxation experiments were conducted on a relaxometer whose description has been given in a previous paper [1]. For the creep and recovery experiment we first allowed the sample to creep for a predetermined amount of time by attaching a known weight to the bottom clamp of the sample. We then cut the sample at a place very near the bottom clamp in order to assure an almost instantaneous release of force on the sample and measured the recovery. The constant rate of strain measurements were obtained on an "Instron" machine. 
For creep, recovery and constant rate of strain measurements, the motions of two marks on the sample, initially $10 \mathrm{~cm}$ apart, were obtained with the aid of two cathetometers. The reference marks were placed far enough away from the clamps to insure that end effects would not disturb the measurements. During the experiments two persons, one at each cathetometer, took readings and a third person recorded the times. Through the use of graphical interpolation, we were able to ascertain accurately the deformation of the sample.

\section{Results and Discussion}

It has been reported previously by Bernstein, Kearsley, and Zapas [1] that the reduced stress for simple extension measurements can be represented very well by the following equation

$$
H(\lambda, t) \equiv \sigma_{R}(\lambda, t)=\alpha(t)\left(\lambda^{2}-1\right)+\frac{1}{\lambda} \beta(t)+\gamma(t) .
$$

Although we may obtain this equation by assuming a particular form of the potential function $U$, we shall regard eq (6) as an empirical equation which describes the surface $H(\lambda, t)$. Thus, for the sake of convenience, without committing ourselves to any particular form of $U_{,}^{3}$ we shall henceforth present and calculate our data with the three material properties $\alpha(t), \beta(t)$, and $\gamma(t)$ of eq (6). Values of these functions are given in tables 1 and 2. In order to describe the data on plas-

TABle 1. Polyisobutylene $L-100$ at $25^{\circ} \mathrm{C}$

\begin{tabular}{r|c|c}
\hline \hline Time & $\beta(t) \times 10^{-6}$ & $\gamma(t) \times 10^{-5}$ \\
\hline & & \\
$\min$ & Dynes $/ \mathrm{cm}^{2}$ & Dynes $/ \mathrm{cm}^{2}$ \\
2 & 1.76 & 6.3 \\
4 & 1.68 & 5.1 \\
0 & 1.63 & 4.45 \\
10 & 1.50 & 3.75 \\
20 & 1.29 & 3.00 \\
40 & 1.064 & 2.38 \\
60 & 0.940 & 2.1 \\
100 & .770 & 1.75 \\
120 & .70 & 1.65 \\
\hline
\end{tabular}

TABLE 2. Plasticized polyvinylchloride at $27{ }^{\circ} \mathrm{C}$

\begin{tabular}{c|c|c|c}
\hline \hline Time & $\alpha(t) \times 10^{-7}$ & $\beta(t) \times 10^{-7}$ & $-\gamma(t) \times 10^{-7}$ \\
\hline & & & \\
$\min$ & Dynes $/ \mathrm{cm}^{2}$ & Dynes $/ \mathrm{cm}^{2}$ & Dynes $/ \mathrm{cm}^{2}$ \\
0.6 & 2.53 & 5.28 & 4.36 \\
1.0 & 2.38 & 4.89 & 4.01 \\
2.0 & 2.21 & 4.61 & 3.64 \\
4.0 & 2.08 & 4.39 & 3.54 \\
6.0 & 2.02 & 4.29 & 3.45 \\
10.0 & 1.94 & 4.17 & 3.34 \\
& & & \\
20.0 & 1.85 & 4.04 & 3.23 \\
40.0 & 1.78 & 3.96 & 3.13 \\
60.0 & 1.74 & 3.85 & 3.07 \\
100.0 & 1.70 & 3.77 & 3.01 \\
120.0 & 1.68 & 3.74 & 2.99 \\
& & & \\
\hline
\end{tabular}

\footnotetext{
${ }^{3}$ Recently a method of correlating results of experiments in biaxial extension and vis-
cometric flow has been presented which utilizes an empirically determined $U$ with three cometric flow has been
material properties $[4 \mid$.
}

ticized PVC we needed all the three material functions. For the polyisobutylene, however, only two were needed. This is in agreement with previous work [1].

\subsection{Double Step}

Suppose that in a two step stress relaxation we take the first step at time $t=0$ with extension ratio $\lambda$ and the second step at $t=t_{1}$, with extension ratio $\eta$, where the extension ratios are taken with respect to the undeformed state. From eqs (4) and (5) we obtain for times greater than $t_{1}$

$$
\begin{aligned}
\sigma(t)= & \left(\dot{\eta}^{2}-\frac{1}{\eta}\right)\{H(\eta, t) \\
& \left.+\frac{\eta^{3}-\lambda^{3}}{\lambda^{2}\left(\eta^{3}-1\right)}\left[H\left(\frac{\eta}{\lambda}, t-t_{1}\right)-H\left(\frac{\eta}{\lambda}, t\right)\right]\right\}
\end{aligned}
$$

In figures 2, 3, and 4 we show the results of two step stress-relaxation experiments for various values of $t_{1}$ and the ratio of $\eta / \lambda$. The data are plotted taking as zero time the time of each step. This is done to emphasize the results at early times after the steps which are the more critical data. The black points represent the calculated values as obtained using eqs (7) and (5). The agreement is excellent.

\subsection{Triple Step}

Consider now a triple step stress relaxation with steps at times $0, t_{1}, t_{2}$ and extension ratios $\lambda, \eta, \xi$, respectively. For times $t>t_{2}$ we obtain from eq (4)

$$
\begin{aligned}
& \sigma(t)=\left(\xi^{2}-\frac{1}{\xi}\right) H(\xi, t)+\left(\frac{\xi^{2}}{\lambda^{2}}-\frac{\lambda}{\xi}\right)\left\{H\left(\frac{\xi}{\lambda}, t-t_{1}\right)\right. \\
& \left.-H\left(\frac{\xi}{\lambda}, t\right)\right\}+\left(\frac{\xi^{2}}{\eta^{2}}-\frac{\eta}{\xi}\right)\left\{H\left(\frac{\xi}{\eta}, t-t_{2}\right)\right. \\
& \left.-H\left(\frac{\xi}{\eta}, t-t_{1}\right)\right\} \text {. }
\end{aligned}
$$

In figure 5, the results of a triple step experiment are shown plotted against the time after each step. The black points represent the calculated values obtained by using eq (5) for the first step, eq (7) for the second step, and eq (8) for the third step. The agreement is very satisfactory. The 3-to 4-percent difference at the early times can be attributed to experimental difficulties. Indeed, because of these difficulties, we know the early time behavior for single step stress relaxation only to within a few percent, and it was these data that were used in calculating our predicted values. The loading time, though small (about 1 sec), may vary by a factor of two, and the manner in which the deformation is applied resembles a very fast ramp function rather than a step function. The temperature change at the early times varies with extension ratio. These effects could contribute significantly at early times after the steps. 


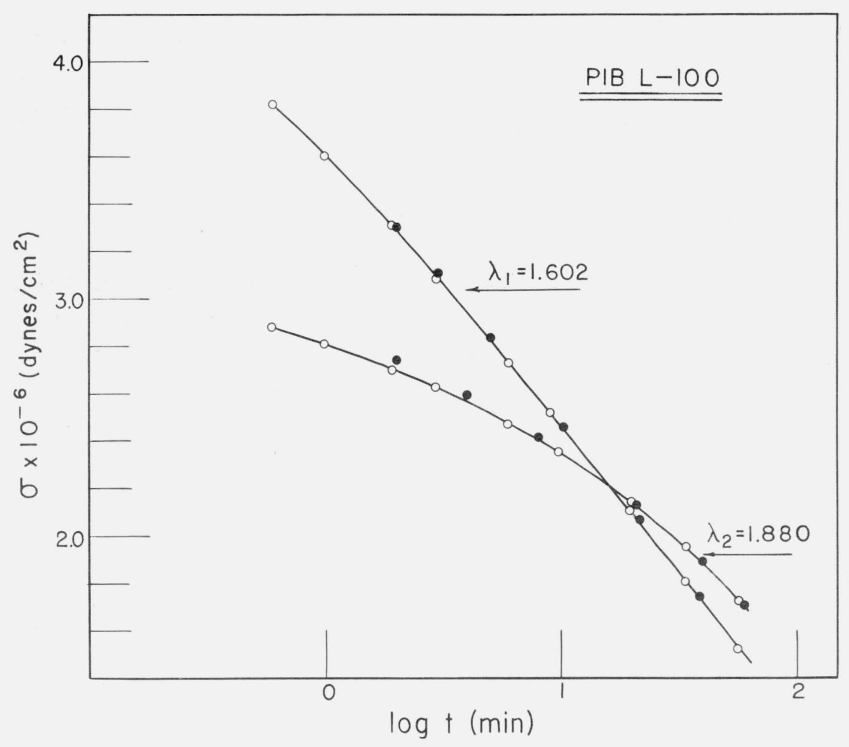

Figure 2. Double step stress relaxation at $25^{\circ} \mathrm{C}$.

First step with extension ratio $\lambda_{1}=1.602$ from 0 min to $60 \mathrm{~min}$. Second step with extension ratio $\lambda_{2}=1.880$ from $60 \mathrm{~min}$ to $130 \mathrm{~min}$.

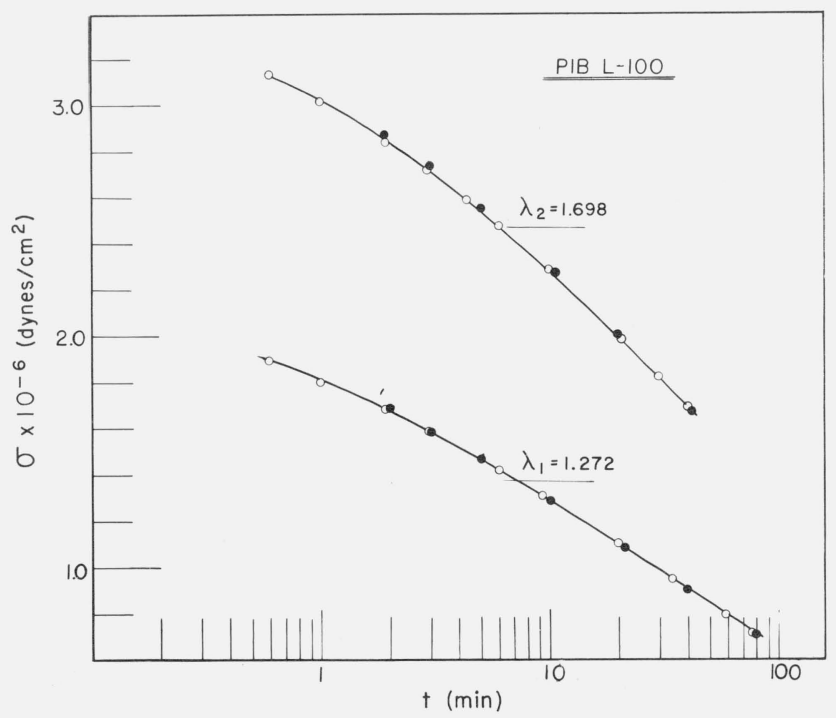

Figure 3. Double step stress relaxation at $25^{\circ} \mathrm{C}$.

First step with extension ratio $\lambda_{1}=1.272$ from $0 \mathrm{~min}$ to $80 \mathrm{~min}$. Second step with extension ratio $\lambda_{2}=1.698$ from $80 \mathrm{~min}$ to $125 \mathrm{~min}$.

\subsection{Creep and Recovery}

In a creep experiment a constant stress or a constant load (as in our experiment) is applied; and the resulting deformation is measured as a function of time. A prediction of the deformation with respect to time requires an inversion of eq (1) or, for the case of simple extension, eq (4). Because this is an arduous task even with the aid of a computer, we elected to check our

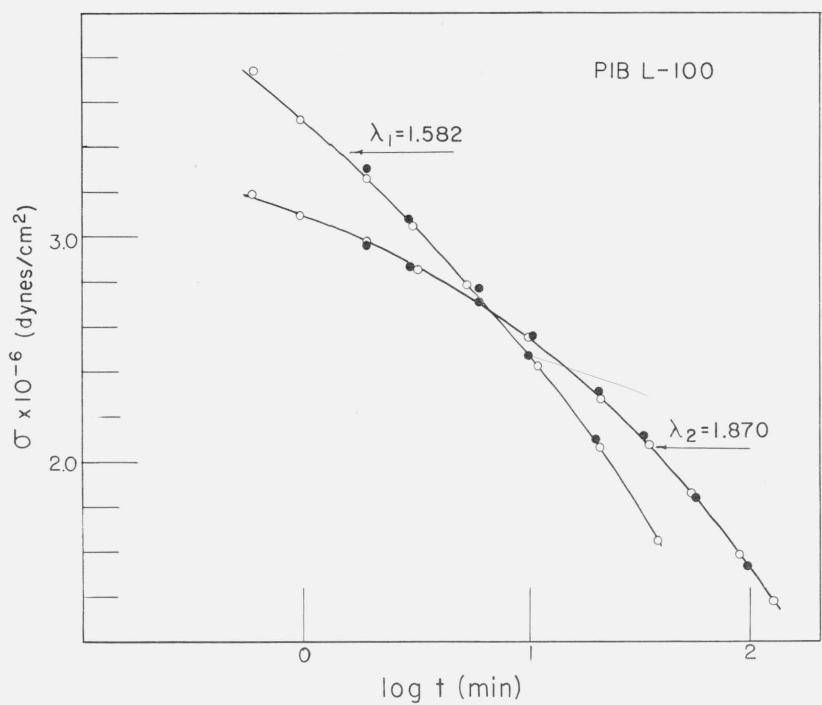

Figure 4. Double step stress relaxation at $25^{\circ} \mathrm{C}$. First step with extension ratio $\lambda_{1}=1.582$ from $0 \mathrm{~min}$ to $40 \mathrm{~min}$. Second step with extension ratio $\lambda_{2}=1.870$ from $40 \mathrm{~min}$ to $165 \mathrm{~min}$.

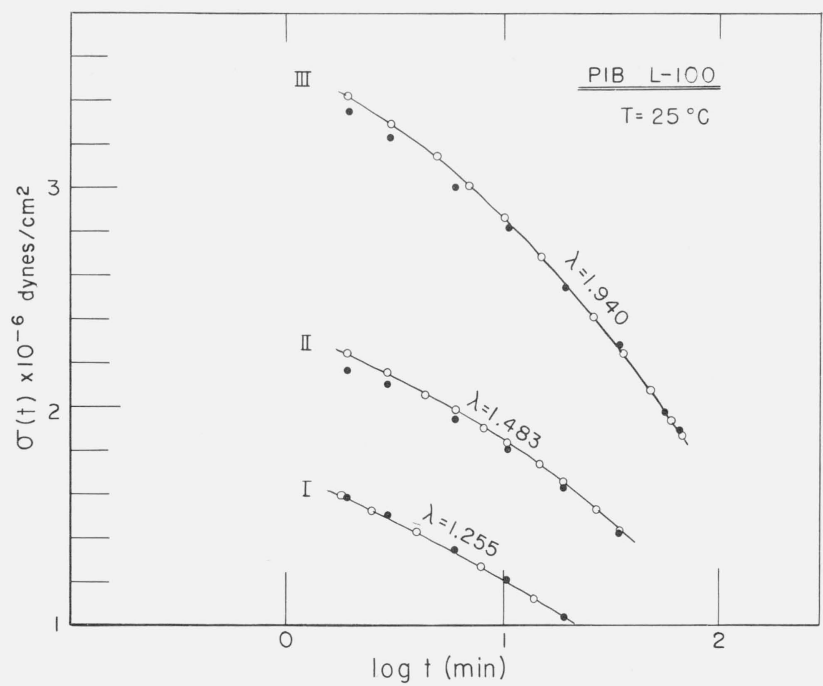

Figure 5. Triple step stress relaxation at $25^{\circ} \mathrm{C}$.

First step with extension ratio $\lambda=1.255$ from 0 min to $20 \mathrm{~min}$. Second step with extension ratio $\lambda=1.483$ from $20 \mathrm{~min}$ to $60 \mathrm{~min}$. Third step with extension ratio $\lambda=1.940$ from $60 \mathrm{~min}$ to $125 \mathrm{~min}$.

results by putting into the right-hand side of eq (4) the measured deformation history up to time $t$ and calculating the stress.

Equation (4) can be written as

$$
\begin{array}{r}
\sigma(t)=\left[\lambda^{2}(t)-\frac{1}{\lambda(t)}\right] H(\lambda, t)+\int_{0}^{t}\left[\frac{\lambda^{2}(t)}{\lambda^{2}(\tau)}-\frac{\lambda(\tau)}{\lambda(t)}\right] \\
h\left(\frac{\lambda(t)}{\lambda(\tau)}, t-\tau\right) d \tau .
\end{array}
$$




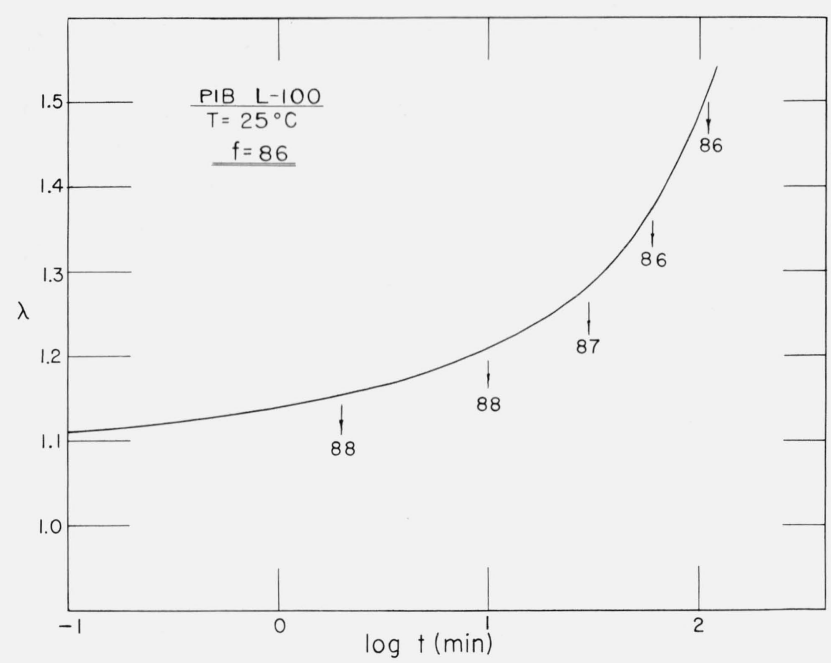

FIGURE 6. Creep of polyisobutylene $L-100$ at $25^{\circ} \mathrm{C}$.

The arrows point to the values of the load calculated using the measured history. The actual load was $86 \mathrm{~g}$.

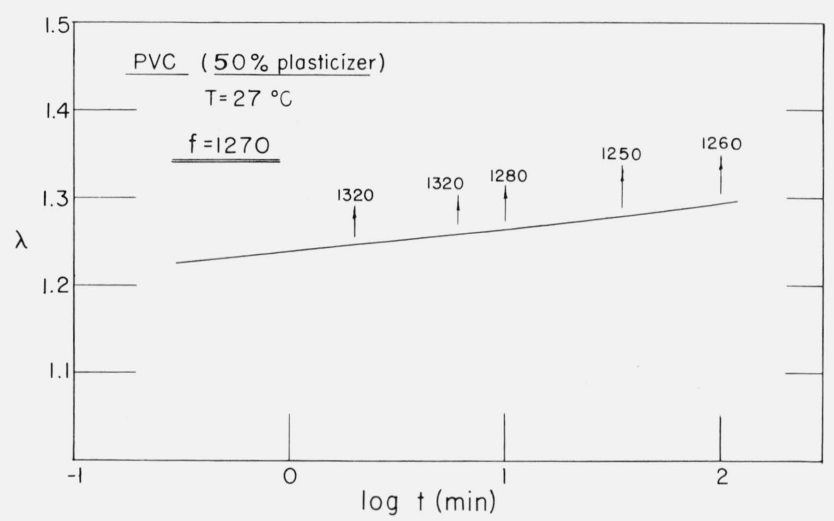

FigURE 7. Creep of plasticized polyvinylchloride at $27^{\circ} \mathrm{C}$. The arrows are used as in figure 6 . The actual load was $1270 \mathrm{~g}$.

The first term of the right-hand side of this equation is the stress necessary to hold a material at an extension ratio $\lambda$ at time $t$ after a single step deformation (vide eq (5)). The second term may be taken as a correction for deviations of the history of the deformation from that of a single step stress-relaxation experiment.

In figure 6 and figure 7 we exhibit the results of creep experiments on polyisobutylene and plasticized PVC. The line shows the value of $\lambda$ as a function of time interpolated from direct measurements. The arrows point to values of the load predicted by eq (9) and the measured history of $\lambda$ up to the indicated tımes. For PIB L-100 the measured load was $86 \mathrm{~g}$ and at the early times we again observe a 2-percent difference from the predicted load, while at the later times the agreement is excellent. The same is observed in figure 7 for plasticized PVC, for which the measured load was $1270 \mathrm{~g}$. Again, this is to be expected, since we do not know $H(\lambda, t)$ accurately at the early times.

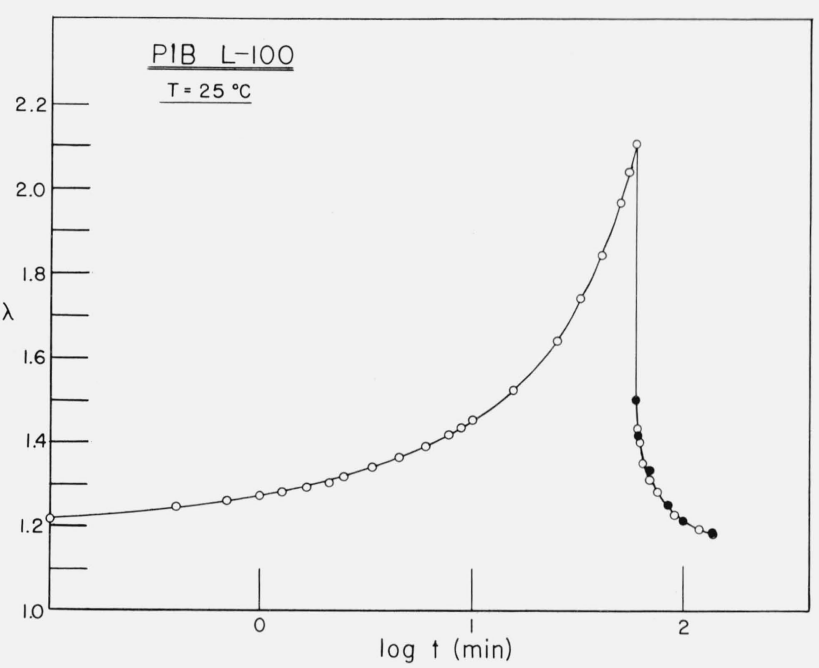

FIGURE 8. Creep and recovery of polyisobutylene at $25^{\circ} \mathrm{C}$. The black circles indicate calculated values. White circles represent measured values.

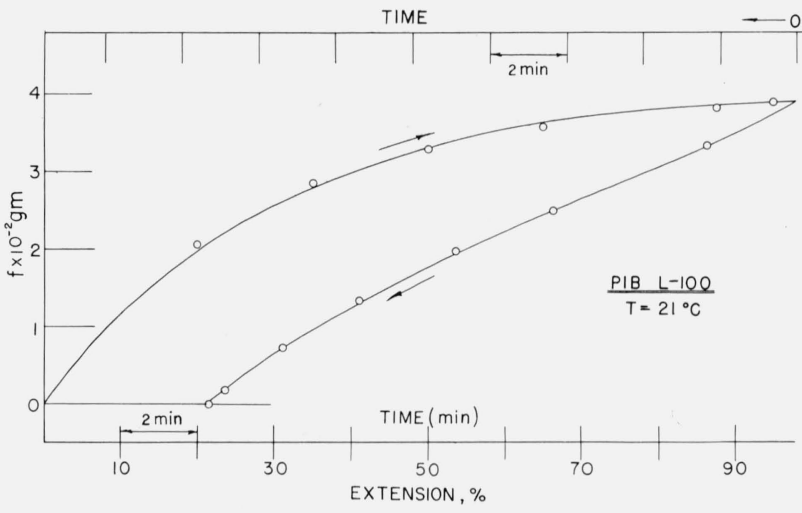

Figure 9. Constant rate of strain of polyisobutylene at $21{ }^{\circ} \mathrm{C}$. The circles represent calculated points.

During the recovery after creep, the part of the history for which $\lambda(t)<\lambda(\tau)$ may be considered as a relative compression. Since the part of the surface $H(\lambda, t)$ that we measured from stress relaxation involved only extensions with $\lambda>1$, we made the assumption in our recovery calculations that eq (6) could be used to describe $H(\lambda, t)$ for values of $\lambda<1$. In figure 8 we show the data obtained on creep and recovery. The black points are values of $\lambda$ during recovery, calculated from the measured values of $\lambda$ prior to recovery using eq (9) with $\sigma$ set equal to zero.

\subsection{Constant Rate of Strain}

In experiments of this type, one of the clamps holding the sample is moved at a constant speed, while the other remained stationary. If there were no end effects due to clamping, the sample would be deformed at a rate proportional to the rate of separation of the clamps. In the experiment whose results we show in figure 9 , we observed a constant rate of strain different 
from what would be predicted by ignoring end effects. The open circles represent values of the stress calculated from eq (9). Here again, in order to predict the part of the curve where the motion is reversed, we made the same assumptions on extrapolation of $H(\lambda, t)$ to the $\lambda$ region as we did for the creep recovery. Further, these data were obtained at a lower temperature than the stress-relaxation data., In order to correct for this temperature difference, we adjusted the stress-relaxation data using time-temperature superposition appropriate to the BKZ elastic fluid [2]. The $H(\lambda, t)$ surfaces at absolute temperatures $T_{0}$ and $T_{1}$ are related as follows ${ }^{4}$

$$
T_{0}^{-1} \rho_{0}^{-1} H(\lambda, t)_{T_{0}}=T_{1}^{-1} \rho_{1}^{-1} H\left(\lambda, b_{T} t\right)_{T_{1}}
$$

where $\rho_{0}$ and $\rho_{1}$ are the densities at $T_{0}$ and $T_{1}$, and $b_{T}$ is a time shifting factor which can either be obtained experimentally or estimated from semiempirical equations. From previously reported work on polyisobutylene by Leaderman [5] the shifting factor $b_{T}$ was evaluated to be 1.45 for a shift from $25{ }^{\circ} \mathrm{C}$ to $21^{\circ} \mathrm{C}$.

The experimental data and the calculated values shown in figure 9 are in excellent agreement. For creep, recovery, and constant rate of strain the calculation of each point involved graphical integration of the expression

$$
\int_{0}^{t}\left[\frac{\lambda^{2}(t)}{\lambda^{2}(\tau)}-\frac{\lambda(\tau)}{\lambda(t)}\right] h\left(\frac{\lambda(t)}{\lambda(\tau)}, t-\tau\right) d \tau,
$$

which is the second term of the right-hand side of eq (9). For values of $\tau$ close to $t$ we used values of $h(\lambda, t)$ extrapolated to small values of $t$. In most of our calculations the first factor in the integrand approached zero as $\tau$ approached $t$, so that we expect the error due to extrapolation to be unimportant.

\footnotetext{
4 The validity of this relation for large deformations has been tested and will be presented in a forthcoming publication.
}

The excellent agreement between the calculated and experimental values for several quite different time histories may be taken as establishing the form of the nonlinear dependence of stress on strain history given by eq (4) for elastomers in the rubbery region. The results reported here, being confined to simple extension, cannot be taken as establishing the potential function, $U$. They should be considered only as a test of the adequacy of the form in which time effects are brought into the BKZ theory. The development of a potential function, $U$, adequate for the description of different geometries of deformation will require detailed studies of different types of deformations.

The authors thank B. Bernstein and E. A. Kearsley for their criticism and suggestions on the preparation of this manuscript.

\section{References}

[1] B. Bernstein, E. A. Kearsley, and L. J. Zapas, A study of stress relaxation with finite strain, Trans. Soc. Rheology VII, 391-410 (1963).

[2] B. Bernstein, E. A. Kearsley, and L. J. Zapas, Thermodynamics of perfect elastic fluids, J. Res. NBS 68B, No. 4, 103-113 (1964).

[3] B. Bernstein, Time-dependent behavior of an incompressible elastic fluid-Some homogeneous deformations, to be published in Acta Mechanica.

[4] L. J. Zapas, Correlations of an elastic fluid with experiments, paper presented at the Society of Rheology meeting. Pittsburgh, October 1964.

[5] H. Leaderman, J. Polymer Sci. 13, 371 (1954).

(Paper 69A6-377) 